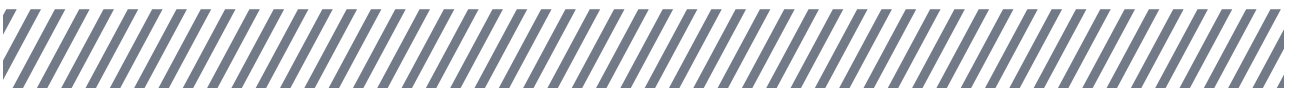

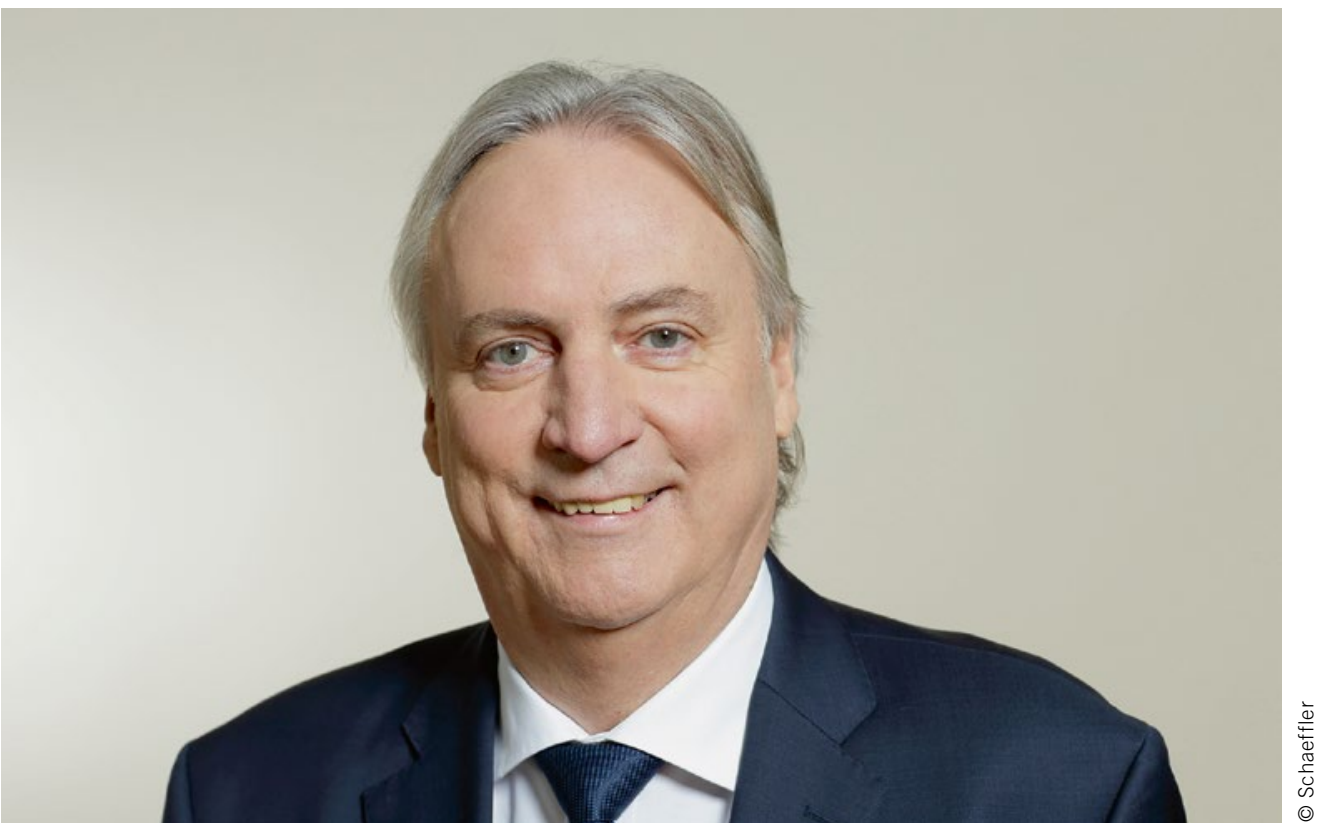

Peter Gutzmer

stellvertretender Vorstandsvorsitzender und Technologievorstand Schaeffler AG

\section{Schlüsselkonzept für die Mobilität von morgen}

Erinnern Sie sich? Der gute, alte Käfer hatte noch ein 6-VStromnetz, bevor sich die 12-V-Bordnetzspannung durchsetzte. Heute stellen Komfortfeatures wie elektrische Heizund Verstelltechniken, Multimediaanlagen, Fahrerassistenzsysteme und insbesondere die zunehmende Elektrifizierung des Antriebs immer höhere Anforderungen an die Stromversorgung. Bauraum und Gewicht der Kabelbäume setzen den weiter steigenden Leistungsbedarfen Grenzen.

Vervierfacht man die Spannung von 12 auf 48 V, lassen sich höhere Leistungen verwirklichen ohne die schärferen Sicherheitsanforderungen der Hochvolttechnik erfüllen zu müssen. Schaeffler hat bereits seit 2010 48-V-Konzepte und -Komponenten für Antrieb und Fahrwerk mit Partnern entwickelt. Die funktionale Erweiterung des 48-V-10-kW-Generators in P0-Position auf Rekuperieren und Segeln zeigt bereits über $15 \% \mathrm{CO}_{2}$-Reduktionspotenzial. Zudem konnte die gesamtsystemische Betrachtung hier auch den Beitrag von E-Clutch-Systemen zur kostengünstigen Elektrifizierung von Schaltgetrieben in Verbindung mit 48-V-Hybridisierung aufzeigen.

Aktuell ist zu erwarten, dass sich in den kommenden Jahren riemenbasierte 48-V-P0-Systeme weltweit als kostengünstige milde Hybridisierung durchsetzen werden. Die milde Hybridisierung bietet aber noch mehr: Schaeffler hat inzwischen weitere Konzepte mit konzentrischer und achsparalleler P2-Anordnung sowie auch als P3- und P4-
Anordnung für erweiterte Rekuperations- und Fahrfunktionen entwickelt und erprobt.

Wir erwarten, dass insbesondere die sehr kompakten gewichtsoptimierten, konzentrischen P2-Module mit den höheren Leistungen im Stadtbetrieb die heutigen Hochvolthybride ersetzen können. Vergleichbares gilt auch für die 48-V-E-Achse. Wir arbeiten gerade daran, diese Lösungen für Serienanwendungen reif $\mathrm{zu}$ machen und ein $\mathrm{CO}_{2}$-Reduktionspotenzial von $20 \mathrm{~g} / \mathrm{km}$ und mehr zu erschließen.

Ein weiteres Anwendungsfeld der 48-V-Technologie liegt im Bereich der elektromechanischen Aktorik. Als erster Sytempartner haben wir gemeinsam mit Porsche, Bentley und Audi einen 48-V-Wankstabilisator in Serie eingeführt und damit Agilität und Fahrkomfort von SUV- und Sport-Limousinen-Plattformen auf ein neues Level gehoben, bei gleichzeitiger Reduktion des Verbrauchs von 3 bis 5 \% gegenüber bekannten hydraulischen Lösungen. Die 48-V-Lösung basiert hier auf einem gemeinsamen Baukasten, der zuerst in den Markt gebrachten 12-V-Standardlösung.

Getrieben durch die Erfordernisse der Antriebs- und Fahrwerksregel-Technologien gehört die Zukunft dem 48-V-Bordnetz, gemeinsam mit dem 12-V-Standard- und Infotainmentnetz und den Hochvoltnetzen für Plug-in-Hybride und E-Fahrzeuge.

Von solchen technischen Raffinessen konnte der Fahrer eines VW-Käfers noch nicht mal träumen. Mit $6 \mathrm{~V}$ waren selbst gute Scheinwerfer eine echte Herausforderung. 